\title{
Estimating Direction of Arrival by Using Two-Dimensional State-Space Balance Method
}

\author{
Jun Wang, Hong Xiang, Shaoming Wei, and Zhongsheng Sun \\ School of Electronic and Information Engineering, Beihang University, Beijing 100191, China \\ Correspondence should be addressed to Shaoming Wei; weishaoming@126.com
}

Received 24 May 2017; Revised 20 September 2017; Accepted 4 October 2017; Published 2 November 2017

Academic Editor: Amerigo Capria

Copyright (c) 2017 Jun Wang et al. This is an open access article distributed under the Creative Commons Attribution License, which permits unrestricted use, distribution, and reproduction in any medium, provided the original work is properly cited.

\begin{abstract}
A study of a two-dimensional state-space balance (2D SSB) method for estimating direction of arrival (DOA) for uniform rectangular array (URA) is presented in this letter. The comprehensive utilization of controllability and observability matrices and automatic pairing technique are considered in this method by using the single snapshot. Therefore, the DOAs of elevation angle and azimuth angle can pair automatically and acquire better estimation performance compared with 2D matrix pencil method or unitary matrix pencil method. In addition, the proposed method can handle correlated signals directly without preprocessing. Simulation is conducted to verify the effectiveness of the proposed method.
\end{abstract}

\section{Introduction}

In array signal processing, target direction of arrival (DOA) estimation has been widely applied in radar, sonar, wireless indoor positioning [1-3] and so on. Since the uniform rectangular array (URA) can provide the information of azimuth and elevation angles, a lot of 2D DOA methods have been developed, such as multiple signal classification (MUSIC) algorithm [4-6], root-MUSIC [7], estimation of signal parameters via rotational invariance technique (ESPRIT) algorithm $[8,9]$, and rank reduce (RARE) algorithm [10]. These conventional high resolution DOA parameter estimation techniques are mainly based on the statistical data by using the covariance matrix under the uncorrelated conditions. In the nonstationary environment, these DOA estimation methods performed poorly without preprocessing, but the matrix pencil (MP) method [11] got good performance by processing the received data directly based on the single snapshot. The unitary matrix pencil (UMP) method [12] can reduce the computation compared with the MP method by using a unitary matrix transformation, which can convert the complex operations to the real operations. The accuracy of both methods is nearly the same. The MP or UMP methods can also be regarded as a statespace method via observability or controllability processing. However, the method that using both observability and controllability matrices was not considered to estimate the DOAs in the spatial domain which may be acquire a good performance. On the other hand, the additional search of the angle pairing is essential to the MP/UMP method by maximizing the cost function, which exploits the orthogonal property between signal subspace and noise subspace. However, the computational complexity of the MP/UMP method is high, and the angle pairing is always mismatched in the low SNR condition. Therefore, many automatic pairing techniques were presented to solve this problem, such as simultaneous diagonalization $[13,14]$, simultaneous Schur decomposition [15], and eigenvalue pairing [16].

This paper presents a two-dimensional state-space balance (2D SSB) method that is applied to the DOA estimation in order to get better DOA estimation than the 2D MP/UMP method. On one hand, the comprehensive utilization of controllability and observability matrices has been considered to improve direction abilities. On the other hand, this method can obtain the elevation angle and azimuth angle without pair matching process via automatic pairing technique.

\section{Signal Model}

The uniform rectangular array (URA) in Figure 1 consists of $M \times N$ identical and omnidirectional sensors with 


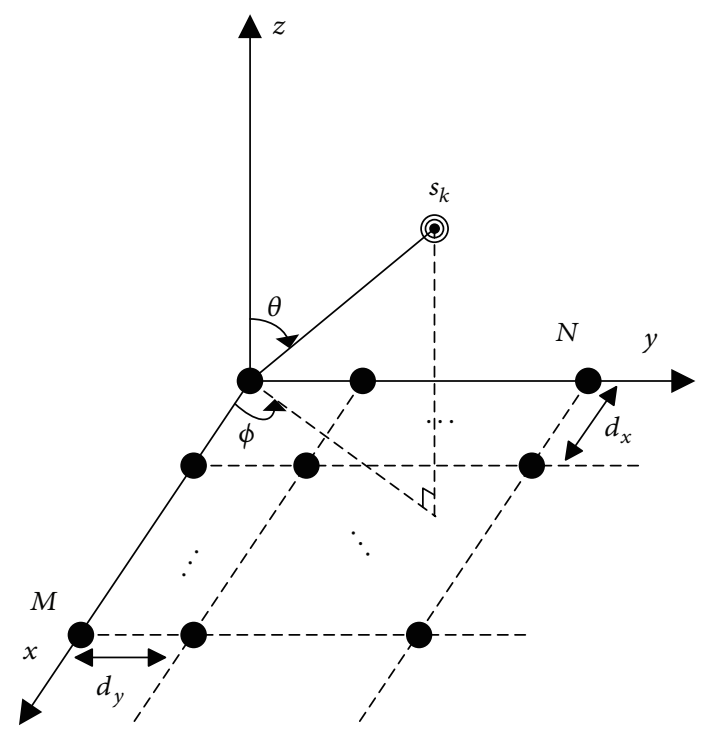

FIGURE 1: Array geometry of the URA.

interelement spacing of $d_{x}=d_{y}=\lambda / 2$, where $\lambda$ is the carrier wavelength.

Suppose $K$ far-field narrowband signals impinge on the URA. $s_{k}$ denotes the $k$ th incident signal, $\theta_{k}$ is the $k$ th elevation angle, and $\phi_{k}$ is the $k$ th azimuth angle, respectively. The received data $z(m, n)$ at the $(m, n)$ th feeding point of the omnidirectional antennas without noise can be expressed as [17]

$$
\begin{aligned}
& z(m, n)=\sum_{k=1}^{K} g_{k} e^{j \pi u_{k} m} e^{j \pi v_{k} n}, \\
& \quad 0 \leq m \leq M-1,0 \leq n \leq N-1,
\end{aligned}
$$

where $u_{k}=\sin \theta_{k} \cos \phi_{k}$ and $v_{k}=\sin \theta_{k} \sin \phi_{k}$ are direction cosines mapping with azimuth and elevation angles and $g_{k}$ is the gain of the $k$ th incident signal.

\section{2D State-Space Balance Method}

Equation (1) can be rewritten in a matrix form akin to that of a state-space impulse response as

$$
\begin{aligned}
& z(m, n)=\mathbf{G} \boldsymbol{\Phi}_{x}^{m} \boldsymbol{\Phi}_{y}^{n} \mathbf{B}, \\
& \qquad 0 \leq m \leq M-1,0 \leq n \leq N-1,
\end{aligned}
$$

where $\boldsymbol{\Phi}_{x}=\operatorname{diag}\left(e^{j \pi u_{1}}, e^{j \pi u_{2}}, \ldots, e^{j \pi u_{K}}\right)$ and $\boldsymbol{\Phi}_{y}=\operatorname{diag}\left(e^{j \pi v_{1}}\right.$, $\left.e^{j \pi v_{2}}, \ldots, e^{j \pi v_{K}}\right)$ are diagonal matrices so that they can commute under state-space system equivalence transformations [18]. $\operatorname{diag}(\cdot)$ denotes the square matrix. $\mathbf{G}=\left[g_{1}, g_{2}, \ldots, g_{K}\right]$ is the gain vector. Diagonal matrices $\boldsymbol{\Phi}_{x}$ and $\boldsymbol{\Phi}_{y}$ contain the DOA information about $u_{k}$ and $v_{k}$. So we will isolate and solve $\Phi_{x}$ and $\Phi_{y}$ by using the 2D SSB method. Firstly, Hankel matrix $\mathbf{D}_{m}$ is formed by using the $m$ th row of the received data array $z(m, n)$ as follows:

$$
\begin{aligned}
& \mathbf{D}_{m} \\
& =\left[\begin{array}{cccc}
z(m, 0) & z(m, 1) & \cdots & z(m, N-C) \\
z(m, 1) & z(m, 2) & \cdots & z(m, N-C+1) \\
\vdots & \vdots & \ddots & \vdots \\
z(m, C-1) & z(m, C) & \cdots & z(m, N-1)
\end{array}\right]
\end{aligned}
$$

where $C$ is the window pencil parameter [17]. We define Hankel matrices $\mathbf{D}_{m}^{+}$and $\mathbf{D}_{m}^{-}$as follows:

$$
\begin{aligned}
& \mathbf{D}_{m}^{+}=\mathbf{D}_{m} \mathbf{J}_{1} \\
& \mathbf{D}_{m}^{-}=\mathbf{D}_{m} \mathbf{J}_{2},
\end{aligned}
$$

where $\mathbf{J}_{1}=\left[\mathbf{I}_{(N-C) \times(N-C)} ; \mathbf{0}_{1 \times(N-C)}\right]$ and $\mathbf{J}_{2}=\left[\mathbf{0}_{1 \times(N-C)}\right.$; $\left.\mathbf{I}_{(\mathrm{N}-\mathrm{C}) \times(\mathrm{N}-\mathrm{C})}\right]$ are selection matrices. $\mathbf{I}_{(\mathrm{N}-\mathrm{C}) \times(\mathrm{N}-\mathrm{C})}$ is identity matrix. Then, Hankel matrices $\mathbf{D}_{m}^{+}$and $\mathbf{D}_{m}^{-}$are used to structure the Hankel block matrices $\mathbf{H}_{00}, \mathbf{H}_{01}$, and $\mathbf{H}_{10}$ as follows:

$$
\mathbf{H}_{00}=\left[\begin{array}{cccc}
\mathbf{D}_{0}^{+} & \mathbf{D}_{1}^{+} & \cdots & \mathbf{D}_{M-B-1}^{+} \\
\mathbf{D}_{1}^{+} & \mathbf{D}_{2}^{+} & \cdots & \mathbf{D}_{M-B}^{+} \\
\vdots & \vdots & \ddots & \vdots \\
\mathbf{D}_{B-1}^{+} & \mathbf{D}_{B-1}^{+} & \cdots & \mathbf{D}_{M-2}^{+}
\end{array}\right]
$$$$
\mathbf{H}_{01}=\left[\begin{array}{cccc}
\mathbf{D}_{0}^{-} & \mathbf{D}_{1}^{-} & \cdots & \mathbf{D}_{M-B-1}^{-} \\
\mathbf{D}_{1}^{-} & \mathbf{D}_{2}^{-} & \cdots & \mathbf{D}_{M-B}^{-} \\
\vdots & \vdots & \ddots & \vdots \\
\mathbf{D}_{B-1}^{-} & \mathbf{D}_{B-1}^{-} & \cdots & \mathbf{D}_{M-2}^{-}
\end{array}\right]
$$

$$
\mathbf{H}_{10}=\left[\begin{array}{cccc}
\mathbf{D}_{1}^{+} & \mathbf{D}_{2}^{+} & \cdots & \mathbf{D}_{M-B}^{+} \\
\mathbf{D}_{2}^{+} & \mathbf{D}_{3}^{+} & \cdots & \mathbf{D}_{M-B+1}^{+} \\
\vdots & \vdots & \ddots & \vdots \\
\mathbf{D}_{B}^{+} & \mathbf{D}_{B+1}^{+} & \cdots & \mathbf{D}_{M-1}^{+}
\end{array}\right] \text {, }
$$

where $B$ is also the window pencil parameter. Substituting (2) and (4) into (5), Hankel matrices $\mathbf{H}_{00}, \mathbf{H}_{01}$, and $\mathbf{H}_{10}$ can be rewritten as

$$
\begin{aligned}
& \mathbf{H}_{00}=\mathbf{O C} \\
& \mathbf{H}_{01}=\mathbf{O} \Phi_{x} \mathbf{C} \\
& \mathbf{H}_{10}=\mathbf{O} \Phi_{y} \mathbf{C},
\end{aligned}
$$

where $\mathbf{O}=\left[\mathbf{G} \boldsymbol{\Phi}_{x}^{0} \boldsymbol{\Phi}_{y}^{0}, \mathbf{G} \boldsymbol{\Phi}_{x}^{1} \boldsymbol{\Phi}_{y}^{0}, \ldots\left|\mathbf{G} \boldsymbol{\Phi}_{x}^{0} \boldsymbol{\Phi}_{y}^{1}, \mathbf{G} \boldsymbol{\Phi}_{x}^{1} \boldsymbol{\Phi}_{y}^{1}, \ldots\right|\right.$ $\ldots]^{\mathrm{T}}$ is the observability matrix, $\mathbf{C}=\left[\boldsymbol{\Phi}_{x}^{0} \boldsymbol{\Phi}_{y}^{0} \mathbf{B}, \boldsymbol{\Phi}_{x}^{1} \boldsymbol{\Phi}_{y}^{0} \mathbf{B}, \ldots\right.$ । $\left.\boldsymbol{\Phi}_{x}^{0} \boldsymbol{\Phi}_{y}^{1} \mathbf{B}, \boldsymbol{\Phi}_{x}^{1} \boldsymbol{\Phi}_{y}^{1} \mathbf{B}, \ldots \mid \ldots\right]$ is the controllability matrix, and $[\cdot]^{\mathrm{T}}$ denotes the transpose. 
Considering the singular value decomposition (SVD) of matrix $\mathbf{H}_{00}$, (6) can be expressed as

$$
\mathbf{H}_{00}=\mathbf{U} \boldsymbol{\Sigma} \mathbf{V}^{\mathrm{H}}=\left[\begin{array}{ll}
\mathbf{U}_{s} & \mathbf{U}_{n}
\end{array}\right]\left[\begin{array}{cc}
\boldsymbol{\Sigma}_{s} & 0 \\
0 & \boldsymbol{\Sigma}_{n}
\end{array}\right]\left[\begin{array}{ll}
\mathbf{V}_{s}^{\mathrm{H}} & \mathbf{V}_{n}^{\mathrm{H}}
\end{array}\right] .
$$

The noise effect should be cut off in order to obtain the high accuracy DOA estimation. Hence, observability matrix $\widetilde{\mathbf{O}}$ and controllability matrix $\widetilde{\mathbf{C}}$ can be acquired as

$$
\begin{aligned}
& \widetilde{\mathbf{O}}=\mathbf{U}_{1: K} \sqrt{\boldsymbol{\Sigma}_{1: K, 1: K}} \\
& \widetilde{\mathbf{C}}=\sqrt{\boldsymbol{\Sigma}_{1: K, 1: K}} V_{1: K}^{\mathrm{H}},
\end{aligned}
$$

where $K$ is the number of signals, which can be adopted by Akaike information criterion (AIC) [19] and minimum description length (MDL) [20]. Then, through the comprehensive utilization of controllability matrix $\widetilde{\mathbf{O}}$ and observability matrix $\widetilde{\mathbf{C}}, \widetilde{\boldsymbol{\Phi}}_{x}$ and $\widetilde{\boldsymbol{\Phi}}_{y}$ can be obtained as

$$
\begin{aligned}
& \widetilde{\boldsymbol{\Phi}}_{x}=\operatorname{pinv}(\widetilde{\mathbf{O}}) \mathbf{H}_{01} \operatorname{pinv}(\widetilde{\mathbf{C}}) \\
& \widetilde{\boldsymbol{\Phi}}_{y}=\operatorname{pinv}(\widetilde{\mathbf{O}}) \mathbf{H}_{10} \operatorname{pinv}(\widetilde{\mathbf{C}}),
\end{aligned}
$$

where $\operatorname{pinv}(\cdot)$ is pseudoinverse. For the implementation simplicity, the eigenvalue pairing [16] technique is applied. Therefore, diagonal matrices $\boldsymbol{\Phi}_{x}$ and $\boldsymbol{\Phi}_{y}$ can be obtained through eigendecomposition of matrices $\widetilde{\boldsymbol{\Phi}}_{x}$ and $\widetilde{\boldsymbol{\Phi}}_{y}$

$$
\begin{aligned}
& \boldsymbol{\Phi}_{x}=\mathbf{T}^{-1} \widetilde{\boldsymbol{\Phi}}_{x} \mathbf{T} \\
& \boldsymbol{\Phi}_{y}=\mathbf{T}^{-1} \widetilde{\boldsymbol{\Phi}}_{y} \mathbf{T},
\end{aligned}
$$

where $\mathbf{T}$ can diagonalize both $\widetilde{\boldsymbol{\Phi}}_{x}$ and $\widetilde{\boldsymbol{\Phi}}_{y}$ in a similarity transformation. Finally, $\left(u_{k}, v_{k}\right)$ pairs can automatically be paired from diagonal matrices $\boldsymbol{\Phi}_{x}$ and $\boldsymbol{\Phi}_{y}$. Therefore, we can get the final results

$$
\begin{aligned}
& \theta_{k}=\arctan \left(\frac{v_{k}}{u_{k}}\right) \\
& \phi_{k}=\arcsin \sqrt{u_{k}^{2}+v_{k}^{2}} .
\end{aligned}
$$

Then the solving steps can be written as follows

Step 1. Construct the Hankel matrices $\mathbf{H}_{00}, \mathbf{H}_{01}$, and $\mathbf{H}_{10}$ from the received data $z(m, n)$.

Step 2. Use the singular value decomposition of Hankel matrix $\mathbf{H}_{00}$ to acquire the left singular vector $\mathbf{U}$, right singular vector $\mathbf{V}$, and singular value $\boldsymbol{\Sigma}$.

Step 3. Construct the controllability matrix $\widetilde{\mathbf{O}}$ and observability matrix $\widetilde{\mathbf{C}}$.

Step 4. Use (11) to obtain matrices $\widetilde{\Phi}_{x}$ and $\widetilde{\Phi}_{y}$;

Step 5. Use eigenvalue pairing technique to get the diagonal matrices $\boldsymbol{\Phi}_{x}$ and $\boldsymbol{\Phi}_{y}$.

Step 6. Use (13) to estimate final results $\left\{\left(\theta_{k}, \phi_{k}\right)\right\}_{k=1}^{K}$.

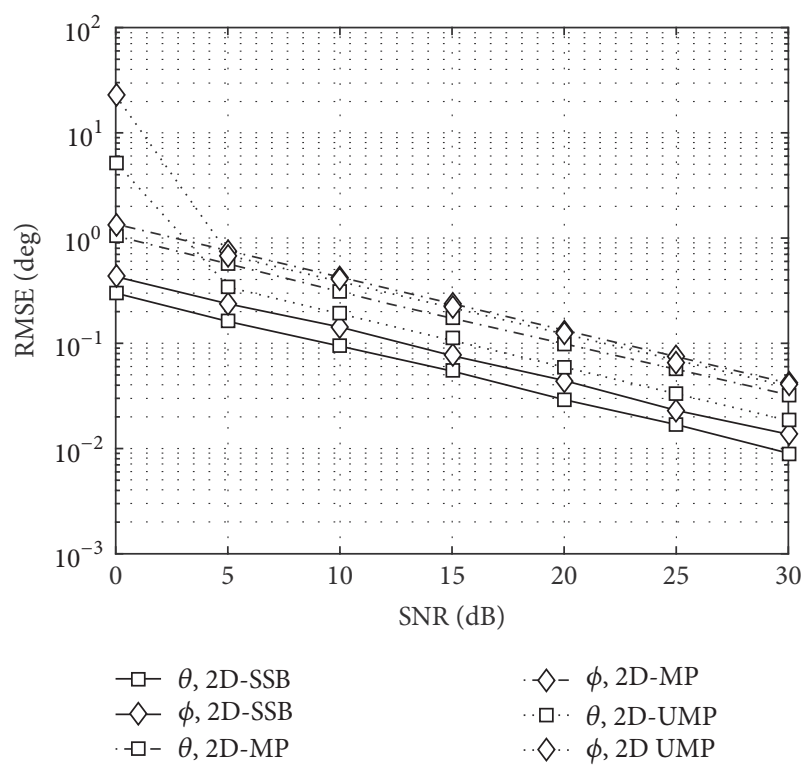

FIGURE 2: RMSEs of azimuth and elevation angles against with SNR for the dispersed sources, where $\left(\theta_{1}, \phi_{1}\right)=\left(25^{\circ}, 55^{\circ}\right)$ and $\left(\theta_{2}, \phi_{2}\right)=$ $\left(45^{\circ}, 65^{\circ}\right)$.

\section{Simulation}

Firstly, two far-field narrowband signals impinge on the $20 \times 20$ URA with $\left(\theta_{1}, \phi_{1}\right)=\left(25^{\circ}, 55^{\circ}\right)$ and $\left(\theta_{2}, \phi_{2}\right)=$ $\left(45^{\circ}, 65^{\circ}\right)$, where the source positions are relatively dispersed. The distance between any two elements of the URA is half a wavelength. The window pencil parameters are $B=10$ and $C=10$. The root mean square errors (RMSEs) of azimuth and elevation angles against with SNR for the URA are illustrated in Figure 2, where 500 Monte Carlo experiments are tested for each data. The RMSEs for the estimates of source DOAs $\theta$ and $\phi$ are defined as

$$
\begin{aligned}
\operatorname{RMSE}_{\theta} & =\sqrt{\frac{1}{J K} \sum_{j=1}^{J} \sum_{k=1}^{K}\left(\widehat{\theta}_{j k}-\theta_{k}\right)^{2}} \\
\operatorname{RMSE}_{\phi} & =\sqrt{\frac{1}{J K} \sum_{j=1}^{J} \sum_{k=1}^{K}\left(\bar{\phi}_{j k}-\phi_{k}\right)^{2}},
\end{aligned}
$$

where $J$ is the number of Monte Carlo experiments. As can be seen from Figure 2, the RMSEs of DOAs $\theta$ and $\phi$ decrease as SNR increases, and the 2D SSB method has a better performance than 2D MP/UMP method [17] at the same SNR, which validates the proposed 2D SSB method.

Secondly, the challenging scenario of closely positioned sources impinging on the same URA is taken into consideration with $\left(\theta_{1}, \phi_{1}\right)=\left(10^{\circ}, 12^{\circ}\right)$ and $\left(\theta_{2}, \phi_{2}\right)=\left(12^{\circ}, 10^{\circ}\right)$. Figure 3 shows the results. The 2D SSB method still has a better performance than the 2D MP/UMP method while the performance of 2D MP method deteriorated sharply even the SNR is very high. In addition, the SNR threshold of $2 \mathrm{D} \mathrm{SSB}$ is much lower than the 2D UMP method. However, in order to acquire a good DOA estimation, a higher SNR threshold is 


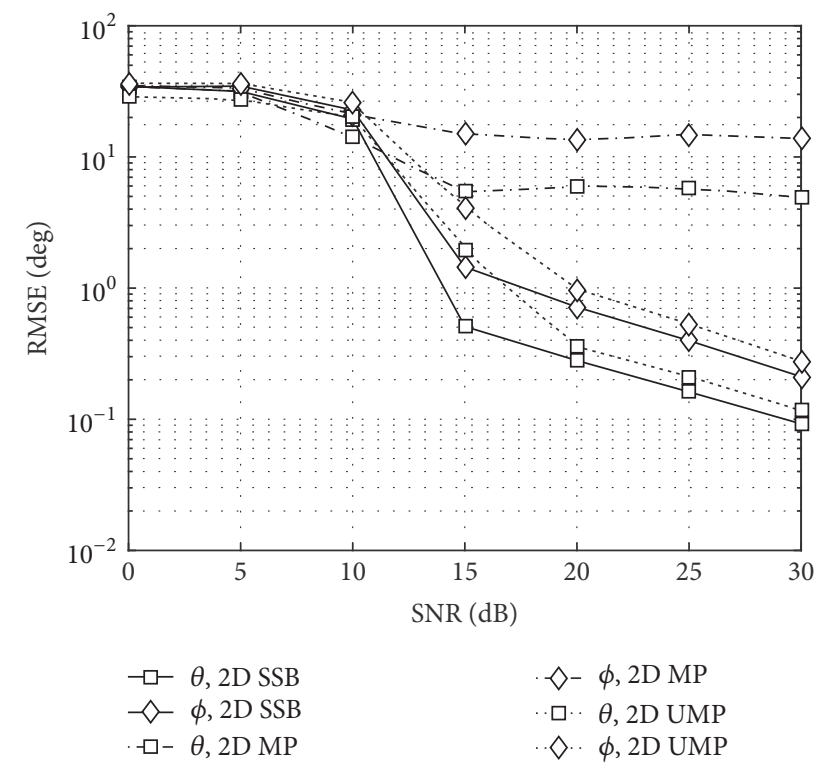

FIGURE 3: RMSEs of azimuth and elevation angles against with SNR for the closely positioned sources, where $\left(\theta_{1}, \phi_{1}\right)=\left(10^{\circ}, 12^{\circ}\right)$ and $\left(\theta_{2}, \phi_{2}\right)=\left(12^{\circ}, 10^{\circ}\right)$.

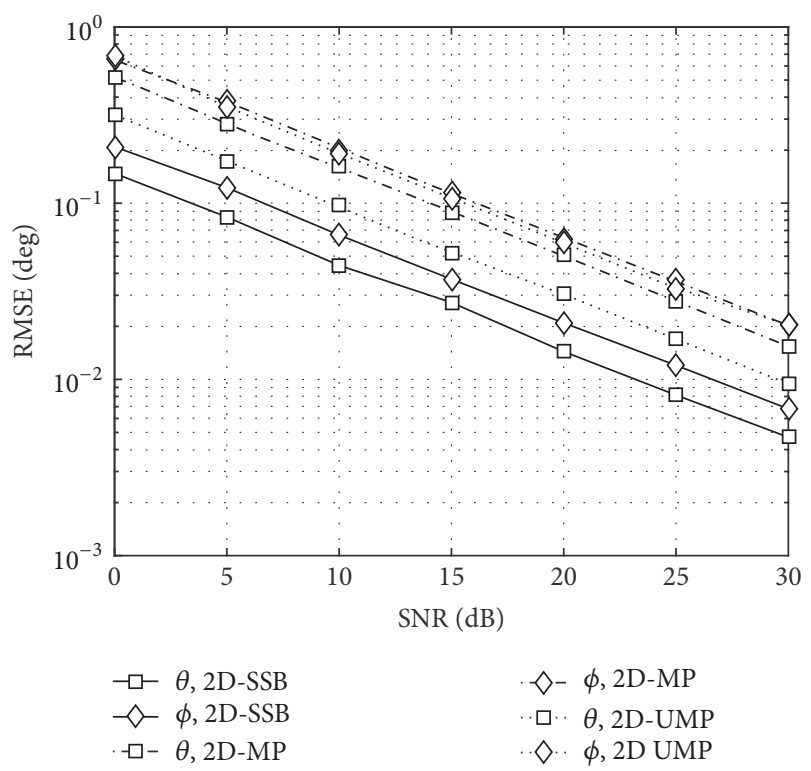

FIGURE 4: RMSEs of azimuth and elevation angles against with SNR for correlated sources, where $\left(\theta_{1}, \phi_{1}\right)=\left(25^{\circ}, 55^{\circ}\right)$ and $\left(\theta_{2}, \phi_{2}\right)=$ $\left(45^{\circ}, 65^{\circ}\right)$.

necessary compared with the scenario in the first simulation. All in all, 2D SSB performs more robustness and effectiveness than 2D MP/UMP method.

Thirdly, same scenario with correlated signals and uncorrelated signals is also taken into consideration in Figures 4 and 5. In order to describe the correlated signals and uncorrelated signals, multiple snapshot case is considered. 2D SSB can easily deal with multiple snapshot case by using the method in [21]. The number of snapshots is set to be
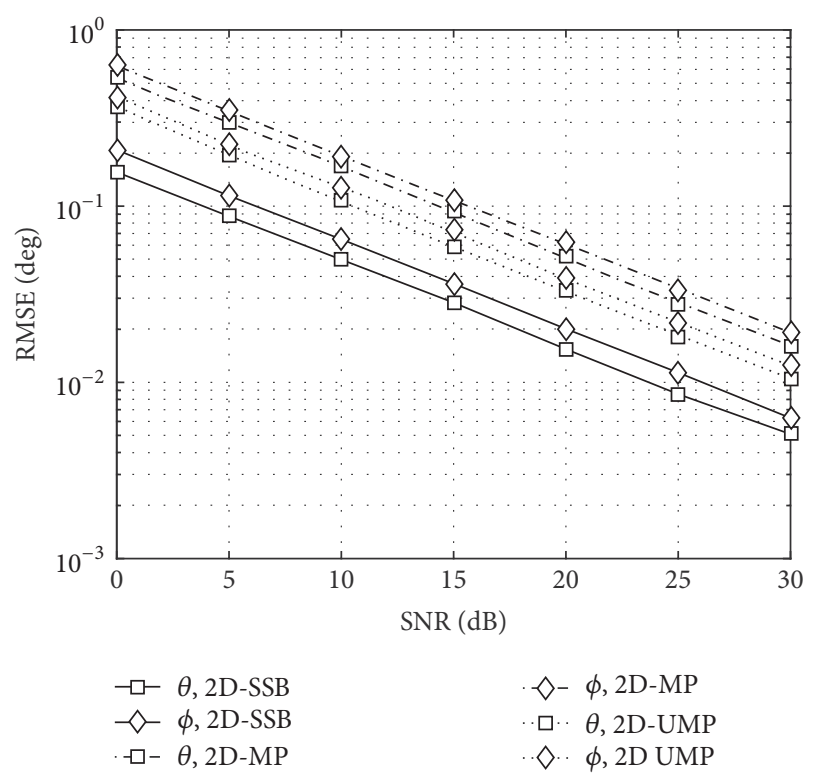

FIGURE 5: RMSEs of azimuth and elevation angles against with SNR for uncorrelated sources, where $\left(\theta_{1}, \phi_{1}\right)=\left(25^{\circ}, 55^{\circ}\right)$ and $\left(\theta_{2}, \phi_{2}\right)=$ $\left(45^{\circ}, 65^{\circ}\right)$.

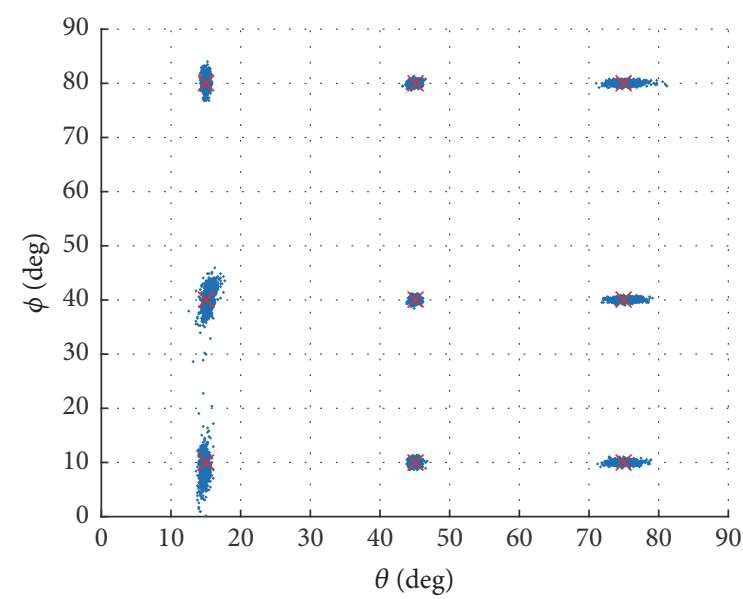

FIGURE 6: 2D DOA estimation results of 9 incident signals, $\mathrm{SNR}=$ $-5 \mathrm{~dB}$.

4. As illustrated in Figures 4 and 5, 2D SSB also performs better.

In the last simulation, 9 incident signals impinge on the same URA. The 2D DOA estimation results for 300 Monte Carlo simulations are shown in Figures 6 and 7 with SNR = $-5 \mathrm{~dB}$ and $\mathrm{SNR}=5 \mathrm{~dB}$, and the estimate values are quite close to the real values indicated by the cross mark. Obviously, the results demonstrate that the angles of azimuth and elevation are paired correctly and effectively. In addition, the proposed method can compute the same azimuths when the elevations are different or the same elevations when the azimuths are different. 


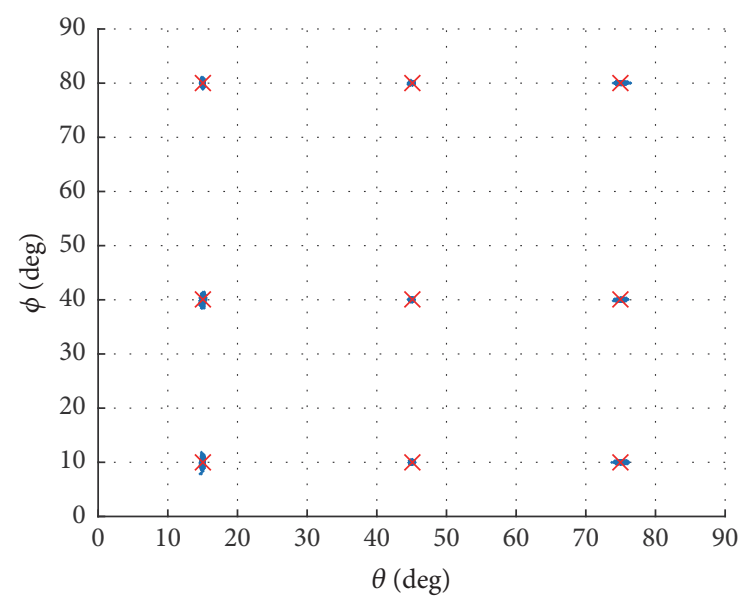

FIGURE 7: 2D DOA estimation results of 9 incident signals, SNR = $5 \mathrm{~dB}$.

\section{Conclusion}

A 2D SSB method is proposed in this paper to estimate the angles of azimuth and elevation. This algorithm indicates high performance by using both observability and controllability matrices. Additionally, this method can provide automatically paired $2 \mathrm{D}$ direction cosine estimates without matching pair and deal with the correlated signals directly. Simulations demonstrate the effectiveness of our method.

\section{Conflicts of Interest}

The authors declare that there are no conflicts of interest regarding the publication of this paper.

\section{Acknowledgments}

This work was supported by the National Natural Science Foundation of China (Grants nos. 61671035 and 61501012).

\section{References}

[1] A. Gaber and A. Omar, "A study of wireless indoor positioning based on joint TDOA and DOA estimation using 2-D matrix pencil algorithms and IEEE 802.11ac," IEEE Transactions on Wireless Communications, vol. 14, no. 5, pp. 2440-2454, 2015.

[2] H. Krim and M. Viberg, "Two decades of array signal processing research," IEEE Signal Processing Magazine, vol. 13, no. 4, pp. 6794, 1996.

[3] M. Y. Cao, S. A. Vorobyov, and A. Hassanien, "Transmit array interpolation for DOA estimation via tensor decomposition in 2-D MIMO radar," IEEE Transactions on Signal Processing, vol. 65, no. 19, pp. 5225-5239, 2017.

[4] R. O. Schmidt, "Multiple emitter location and signal parameter estimation," IEEE Transactions on Antennas and Propagation, vol. 34, no. 3, pp. 276-280, 1986.

[5] F.-G. Yan, Z.-K. Chen, M.-J. Sun, Y. Shen, and M. Jin, “Twodimensional direction-of-arrivals estimation based on onedimensional search using rank deficiency principle," International Journal of Antennas and Propagation, vol. 2015, Article ID 127621, pp. 1-8, 2015.
[6] Y. Dong, C. Dong, W. Liu, H. Chen, and G. Zhao, "2-D DOA Estimation for L-Shaped Array With Array Aperture and Snapshots Extension Techniques," IEEE Signal Processing Letters, vol. 24, no. 4, pp. 495-499, 2017.

[7] A. Ghobadzadeh and R. Adve, "Low-complexity 2D rootMUSIC pairing for an L-shaped array," in Proceedings of the IEEE Radar Conference (RadarConf'17), pp. 0957-0962, Seattle, Wash, USA, May 2017.

[8] R. Roy and T. Kailath, "ESPRIT-estimation of signal parameters via rotational invariance techniques," IEEE Transactions on Signal Processing, vol. 37, no. 7, pp. 984-995, 1989.

[9] Y. Fayad, C. Wang, and Q. Cao, "Improved ESPRIT method used for 2D-DOA estimation in tracking radars," in Proceedings of the 13th European Radar Conference (EuRAD '16), pp. 113-116, London, UK, October 2016.

[10] X. Lan, Y. Li, and E. Wang, "A RARE Algorithm for 2D DOA Estimation Based on Nested Array in Massive MIMO System," IEEE Access, vol. 4, pp. 3806-3814, 2016.

[11] T. K. Sarkar and O. Pereira, "Using the matrix pencil method to estimate the parameters of a sum of complex exponentials," IEEE Antennas and Propagation Magazine, vol. 37, no. 1, pp. 4855, 1995.

[12] N. Yilmazer, J. Koh, and T. K. Sarkar, "Utilization of a unitary transform for efficient computation in the matrix pencil method to find the direction of arrival," IEEE Transactions on Antennas and Propagation, vol. 54, no. 1, pp. 175-181, 2006.

[13] J.-F. Cardoso and A. Souloumiac, "Jacobi angles for simultaneous diagonalization," SIAM Journal on Matrix Analysis and Applications, vol. 17, no. 1, pp. 161-164, 1996.

[14] Y.-Y. Dong, C.-X. Dong, Z.-B. Shen, and G.-Q. Zhao, “Conjugate augmented spatial temporal technique for 2-D DOA estimation with L-shaped array," IEEE Antennas and Wireless Propagation Letters, vol. 14, pp. 1622-1625, 2015.

[15] M. Haardt and J. A. Nossek, "Simultaneous schur decomposition of several nonsymmetric matrices to achieve," IEEE Transactions on Signal Processing, vol. 46, no. 1, pp. 161-169, 1998.

[16] M. L. Burrows, “Two-dimensional ESPRIT with tracking for radar imaging and feature extraction," IEEE Transactions on Antennas and Propagation, vol. 52, no. 2, pp. 524-532, 2004.

[17] N. Yilmazer and T. K. Sarkar, "2-D unitary matrix pencil method for efficient direction of arrival estimation," Digital Signal Processing, vol. 16, no. 6, pp. 767-781, 2006.

[18] J. H. David, State-Space Approaches to Ultra-Wideband Doppler Processing, Worcester Polytechnic Institute, Worcester, Mass, USA, 2007.

[19] M. Wax and T. Kailath, "Detection of signals by information theoretic criteria," IEEE Transactions on Acoustics, Speech and Signal Processing, vol. 33, no. 2, pp. 387-392, 1985.

[20] M. Wax and I. Ziskind, "Detection of the number of coherent signals by the MDL principle," IEEE Transactions on Signal Processing, vol. 37, no. 8, pp. 1190-1196, 1989.

[21] N. Yilmazer, S. Ari, and T. K. Sarkar, "Multiple snapshot direct data domain approach and ESPRIT method for direction of arrival estimation," Digital Signal Processing, vol. 18, no. 4, pp. 561-567, 2008. 


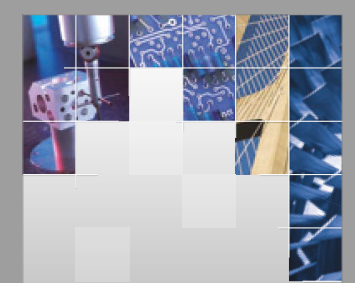

\section{Enfincering}
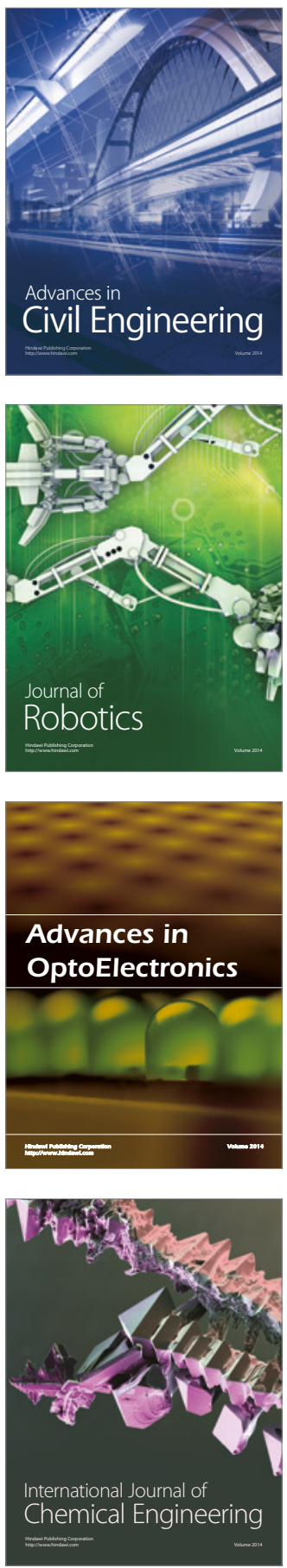

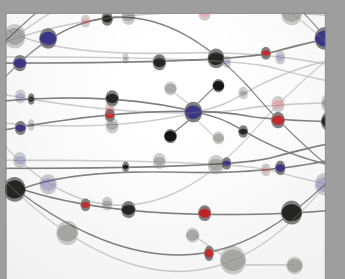

The Scientific World Journal

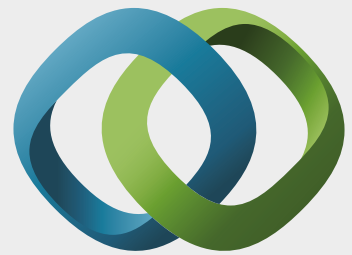

\section{Hindawi}

Submit your manuscripts at

https://www.hindawi.com
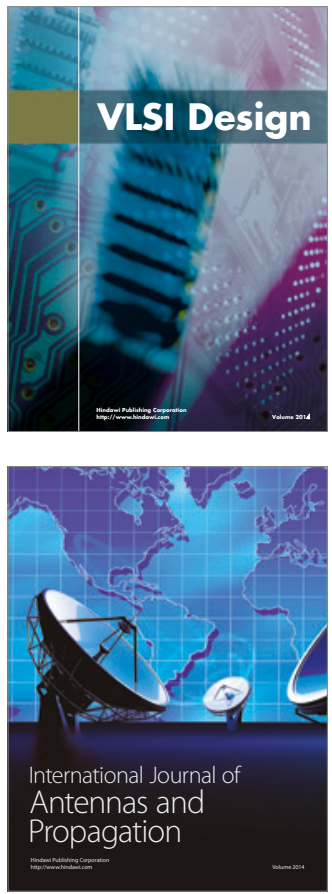

\section{Rotating}

Machinery
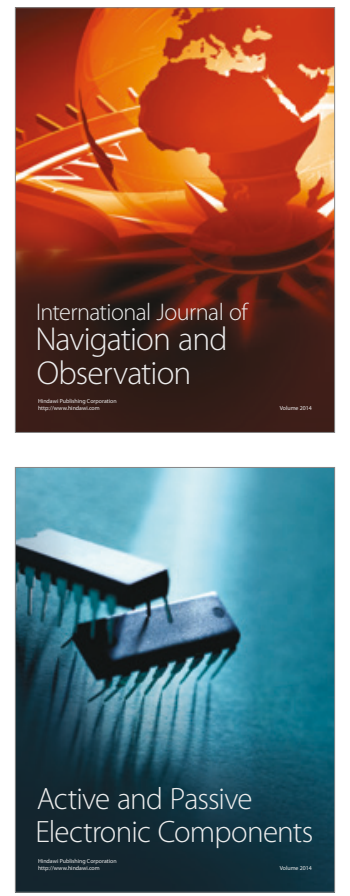
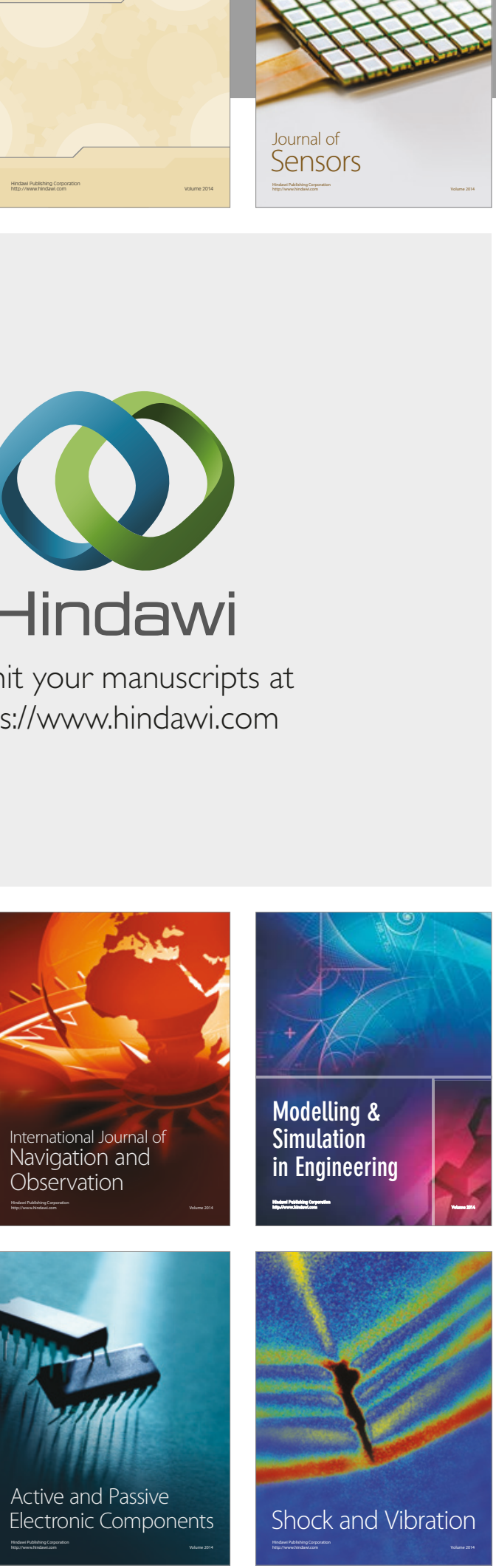
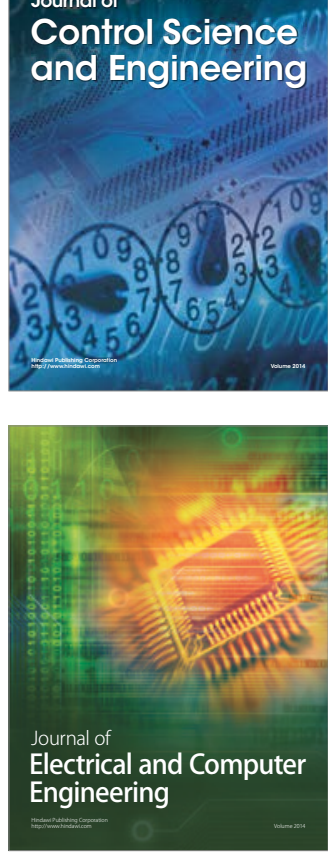

Distributed

Journal of

Control Science

and Engineering
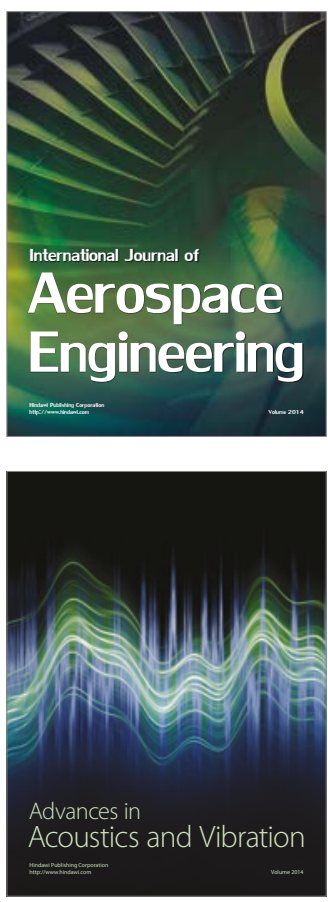

Sensor Networks 